\title{
Avaliação da gestão para o planejamento em saúde em municípios catarinenses
}

\author{
Evaluation of Management for Health Planning in municipalities \\ in the State of Santa Catarina.
}

Josimari Telino de Lacerda ${ }^{1}$

Maria Cristina Marino Calvo ${ }^{1}$

Isabel Quint Berretta ${ }^{1}$

Ângela Maria Blatt Ortiga ${ }^{1}$

${ }^{1}$ Programa de PósGraduação em Saúde Coletiva, Núcleo de Extensão e Pesquisa em Avaliação em Saúde, Universidade Federal de Santa Catarina. Campus Universitário, Centro de Ciências da Saúde sala 106, Trindade. 88040-900 Florianópolis SC. jtelino@gmail.com
Abstract This article presents the results of the evaluation of management for health planning in municipalities in the Southern Brazilian State of Santa Catarina. An evaluation model developed and validated from the theoretical framework for health planning was applied. It was complemented by guidelines for organization and operation of PlanejaSUS (national survey of 2007) and definitions of municipal responsibilities defined in the Management Pact. The evaluation matrix has two dimensions: the guarantee of resources and the guarantee of internal and external relations, with six sub-dimensions and twelve indicators. Data were collected via an electronic form developed by FormSUS, forwarded to and answered by $100 \%$ of the municipalities of Santa Catarina. The "good performance" classification was awarded in 113 municipalities in the overall evaluation, 96 in the dimension of guarantee of resources, and 43 in the dimension of guarantee of relations. The percentage classified as "poor" was high for some indicators, especially human resources and interaction with the population. The results suggest the need to reinforce actions linked to the dimension of guarantee of relations. Key words Health evaluation, Health management, Health planning
Resumo Este artigo apresenta os resultados da avaliação da gestão para o planejamento em saúde em municípios catarinenses. Foi aplicado um modelo de avaliação desenvolvido e validado a partir do referencial teórico de planejamento em saúde, complementado por orientações de organização e funcionamento do PlanejaSUS (pesquisa nacional de 2007) e por definições de responsabilidades municipais preconizadas no Pacto de Gestão. A matriz de avaliação aplicada tem duas dimensões: a garantia de recursos e a garantia de relações internas e externas, com seis subdimensões e doze indicadores. Os dados foram coletados por meio de formulário eletrônico desenvolvido no FormSUS, encaminhado e respondido por 100\% dos municípios catarinenses. A classificação de "bom desempenho" foi observada em 113 municípios na avaliação geral, 96 na dimensão garantia de recursos, e 43 na garantia das relações. Os percentuais com classificação "ruim" foram altos em alguns indicadores, principalmente os de recursos humanos e de articulação com a população. Os resultados sugerem a necessidade de reforçar as ações vinculadas à dimensão de garantia das relações.

Palavras-chave Avaliação em saúde, Gestão em saúde, Planejamento em saúde 


\section{Introdução}

A articulação de um conjunto amplo, e por vezes heterogêneo, de organizações é a estratégia adotada na implementação de políticas na área social frente à complexa rede de causalidade dos problemas e da interface com diferentes atores e setores. É o caso do setor saúde, que agrega diferentes atores, unidades, programas e serviços para garantir o cuidado à saúde de toda a sociedade conformando interesses e conflitos que exigem um grande esforço e muita competência na sua gestão.

O processo de consolidação do Sistema Único de Saúde estimula os gestores a identificarem, selecionarem e elaborarem instrumentos que os auxiliem na condução da gestão. Essa prática contribui para o melhor aproveitamento das oportunidades e para a superação de desafios na implementação de políticas de saúde. A descentralização da gestão é um importante pilar desta consolidação. Concretiza-se pela transferência de serviços, poder, recursos e responsabilidades aos municípios. Uma política substantiva de descentralização e com abertura para o controle social reforça a tendência de reduzir a forma normativa e centralizada da gestão no nível federal e confere aos municípios a direcionalidade que o sistema necessita para produzir saúde. A política de descentralização administrativa pressupõe a criação de estratégias para a superação de entraves gerenciais do setor. Nesse contexto, o planejamento passou a ser considerado como uma importante ferramenta para o efetivo funcionamento do SUS.

O planejamento é um instrumento de gestão das três esferas de governo no âmbito do SUS, permitindo a definição de objetivos e a organização das ações a serem desenvolvidas. Além disso, o planejamento facilita o acompanhamento, a fiscalização, o controle dos gastos e a avaliação dos resultados obtidos. É um instrumento de gestão fundamental para o cumprimento das diretrizes operacionais que orientam o Sistema Único de Saúde e que compõe parte do seu arcabouço legal.

Dentre as várias iniciativas que visam contribuir com o aperfeiçoamento da gestão e a qualificação do planejamento encontram-se o componente Pacto de Gestão do Pacto pela Saúde e a conformação do Sistema de Planejamento do SUS- PlanejaSUS. Ambos tratam da importância da atuação contínua, articulada, integrada e solidária do planejamento nas três esferas de governo, de forma ascendente e orientada pelas necessidades locais; reforçam que o processo de planejamento deve considerar os determinantes e os condicionantes de saúde, promover a partici- pação social e a integração intra e intersetorial; determinam que os pactos das bases funcionais do planejamento se deem de forma tripartite, incluindo o monitoramento e a avaliação; apontam para a necessária adoção de medidas que viabilizem o aprimoramento das atividades, como a definição de métodos e instrumentos de planejamento, monitoramento e avaliação, e provimento de infraestrutura para o desenvolvimento desta atividade.

As orientações definidas pelo PlanejaSUS e pelo Pacto de Gestão avançam para que o planejamento responda efetivamente às necessidades do Sistema de Saúde e às demandas que se impõem continuamente aos gestores. Reforçam a convicção de que a atividade de planejamento deve ter uma participação técnica mais efetiva nas decisões políticas dos gestores da saúde ${ }^{1}$.

$\mathrm{O}$ estabelecimento de responsabilidades de municípios, estados e federação quanto ao planejamento institui a necessidade permanente de informações que favoreçam a reflexão dos gestores sobre os modos de operar as atividades de planejamento e os auxiliem na tomada de decisão para ajustá-las às necessidades do SUS.

O presente estudo realizou a avaliação da eficácia da gestão para as atividades de planejamento nos municípios de Santa Catarina, visando identificar o compromisso da gestão municipal para o desenvolvimento das atividades na área de planejamento em saúde. O objetivo da avaliação proposta é produzir informações que contribuam para a institucionalização e o fortalecimento da área de planejamento nas secretarias municipais de saúde.

\section{Metodologia}

Trata-se de uma avaliação normativa realizada pelo Núcleo de Extensão e Pesquisa em Avaliação em Saúde (NEPAS) da Universidade Federal de Santa Catarina em parceria com a Gerência de Planejamento em Saúde (GEPAS) da Secretaria de Estado da Saúde de Santa Catarina. O critério que orientou a avaliação foi a eficácia da gestão, assumida como a habilidade do gestor em tomar decisões para $o$ atendimento das metas estabelecidas em documentos que norteiam as ações de planejamento em saúde, no âmbito do SUS. Utilizou o modelo de avaliação proposto por Berretta et al. ${ }^{2}$, orientado pelas recomendações e definições de responsabilidade dos municípios quanto ao planejamento definidas nos documentos do PlanejaSUS ${ }^{1}$ e do Pacto de Gestão ${ }^{3}$. 
O modelo aplicado considera duas dimensões avaliativas - Garantia dos Recursos e Garantia das Relações - subdivididas em três subdimensões, com doze indicadores. Para cada medida foram adotados parâmetros normati- vos e/ou indicados na literatura para formular a emissão de juízos de valor em três categorias bom, regular, ruim. As dimensões, subdimensões, indicadores e respectivas medidas estão apresentados no Quadro 1.

Quadro 1. Matriz de Avaliação da Gestão para o Planejamento em Saúde.

\begin{tabular}{|c|c|c|}
\hline \multicolumn{3}{|c|}{ Dimensão: Garantia dos recursos } \\
\hline \multicolumn{3}{|l|}{ Subdimensão: Recursos materiais } \\
\hline $\begin{array}{l}\text { Espaço físico para atividades de } \\
\text { planejamento. }\end{array}$ & \multicolumn{2}{|r|}{ Percepção pelo gestor quanto à adequação do espaço físico. } \\
\hline Materiais de apoio logístico. & \multicolumn{2}{|r|}{$\begin{array}{l}\text { Quantidade de materiais de apoio logístico, dentre os itens } \\
\text { fundamentais, considerados adequados. }\end{array}$} \\
\hline \multicolumn{3}{|l|}{ Subdimensão: Recursos humanos } \\
\hline \multirow{2}{*}{ Vínculo de trabalho. } & \multicolumn{2}{|c|}{$\begin{array}{l}\text { Municípios com } 20 \text { ou menos pessoas na equipe: Número de } \\
\text { profissionais com vínculo não permanente na equipe de planejamento }\end{array}$} \\
\hline & \multicolumn{2}{|c|}{$\begin{array}{l}\text { Municípios com mais de } 20 \text { pessoas na equipe: Percentual de } \\
\text { profissionais com vínculo não permanente na equipe de planejamento }\end{array}$} \\
\hline \multirow{2}{*}{$\begin{array}{l}\text { Permanência de profissionais } \\
\text { na área de planejamento. }\end{array}$} & \multicolumn{2}{|c|}{$\begin{array}{l}\text { Municípios com menos de } 4 \text { pessoas na área de planejamento: } \\
\text { Número de profissionais lotados na área com pelo menos } 5 \text { anos de } \\
\text { atuação em planejamento. }\end{array}$} \\
\hline & \multicolumn{2}{|c|}{$\begin{array}{l}\text { Municípios com } 4 \text { ou mais pessoas na área de planejamento: } \\
\text { Percentual de profissionais lotados na área com pelo menos } 5 \text { anos de } \\
\text { atuação em planejamento }\end{array}$} \\
\hline $\begin{array}{l}\text { Qualificação na área de } \\
\text { planejamento. }\end{array}$ & \multicolumn{2}{|c|}{$\begin{array}{l}\text { Existência de pelo menos um profissional com especialização em } \\
\text { Planejamento ou Saúde Pública. }\end{array}$} \\
\hline \multicolumn{3}{|c|}{ Subdimensão: Recursos de informação } \\
\hline Atualização dos bancos de dados. & \multicolumn{2}{|c|}{ Meses sem registro de informação do SIA e do SIAB } \\
\hline Equipamentos de informática & \multicolumn{2}{|c|}{$\begin{array}{l}\text { Percentual de itens de equipamentos considerados adequados pelo } \\
\text { gestor dentre os itens fundamentais. }\end{array}$} \\
\hline \multicolumn{3}{|c|}{ Dimensão: garantia das relações } \\
\hline \multicolumn{3}{|c|}{ Subdimensão: Articulação intrassetorial } \\
\hline \multicolumn{2}{|l|}{ Inserção na estrutura organizacional. } & $\begin{array}{l}\text { Posição relativa da área de planejamento na estrutura } \\
\text { organizacional da instituição. }\end{array}$ \\
\hline \multicolumn{2}{|c|}{$\begin{array}{l}\text { Articulação entre o planejamento e as } \\
\text { outras áreas técnicas. }\end{array}$} & $\begin{array}{l}\text { Frequência referida quanto ao grau de articulação de } \\
\text { trabalhos entre o planejamento e as outras áreas técnicas. }\end{array}$ \\
\hline \multicolumn{3}{|c|}{ Subdimensão: Articulação intersetorial } \\
\hline \multicolumn{2}{|c|}{$\begin{array}{l}\text { Articulação entre o planejamento e as } \\
\text { outras secretarias }\end{array}$} & $\begin{array}{l}\text { Frequência referida de trabalhos conjuntos entre } \\
\text { planejamento e orçamento. }\end{array}$ \\
\hline \multicolumn{3}{|c|}{ Subdimensão: Articulação com população } \\
\hline Plano Municipal de Saúde & & $\begin{array}{l}\text { Aprovação do Plano Municipal de Saúde pelo Conselho } \\
\text { Municipal de Saúde }\end{array}$ \\
\hline Relatório Anual de Gestão & & $\begin{array}{l}\text { Aprovação do Relatório Anual de Gestão pelo Conselho } \\
\text { Municipal de saúde }\end{array}$ \\
\hline
\end{tabular}


Os dados utilizados foram coletados em 2010, reportando os aspectos da gestão em 2009. Do Relatório de Gestão Municipal de 2009 foram coletados os dados para os indicadores da subdimensão de Articulação com a População; do SIA e SIAB, ano base 2009, foram coletados os dados para o indicador "atualização dos bancos de dados" da subdimensão de Recursos de Informação. Os demais dados foram obtidos em coleta por meio de formulário específico aplicado via online utilizando a ferramenta operacional do FormSUS, no período de 26/07 a 13/11 de 2010, com adesão de $100 \%$ dos municípios catarinenses $(n=293)$.

Promoveu-se a análise descritiva dos dados, classificando indicadores, subdimensões, dimensões e municípios quanto ao alcance dos parâmetros definidos. Todos os indicadores foram considerados com o mesmo grau de importância para emissão de juízo de valor. Foram adotados escores, definindo-se o valor zero para os que não atenderam aos parâmetros estabelecidos, um para os que atenderam parcialmente $\mathrm{e}$ dois para os que atenderam totalmente. Os valores foram agregados por subdimensão e dimensão, como apresentado na Tabela 1 .

Os resultados foram apresentados em tabela, com frequências absolutas e relativas, e em distribuição cartográfica, segundo as dimensões. Possíveis associações com porte populacional, percentual de investimento próprio em saúde, localização geográfica e IDH municipal foram investigadas com teste de qui-quadrado de Pearson.

Os dados utilizados no estudo são de domínio público, não sendo necessária submissão ao Comitê de Ética em Pesquisa.

\section{Resultados}

O desempenho dos municípios catarinenses está apresentado na Tabela 2. A gestão para o planejamento em saúde foi considerada satisfatória na maior parte dos municípios catarinenses: 253 $(86,3 \%)$ municípios apresentaram desempenho bom ou regular.

$\mathrm{Na}$ análise das dimensões observa-se um maior empenho dos municípios na Garantia de Recursos, com aproximadamente um terço classificado como bom desempenho. O detalhamento dessa dimensão permite identificar que os indicadores com melhores resultados em toda a matriz avaliativa foram disponibilização de equipamentos $(81,2 \%)$ e atualização de banco de dados $(77,1 \%)$, ambos compondo a subdimensão $\boldsymbol{R e}$ - cursos de Informação, consequentemente com os melhores resultados na subdimensão - 64,5\% dos municípios apresentou bom desempenho. O segundo melhor desempenho foi observado na subdimensão Recursos Humanos, com quase metade dos municípios classificados como bom. Neste item o indicador permanência de profissionais na área de planejamento apresentou $54,6 \%$ dos municípios classificados como bom desempenho. Os piores desempenhos foram encontrados para espaço físico e materiais de apoio logístico, ambos na subdimensão Recursos Materiais, na qual a maioria dos municípios apresentou desempenho regular e apenas $18,1 \%$ desempenho bom.

$\mathrm{Na}$ análise da dimensão Garantia das Relações, a maioria dos municípios apresentou desempenho regular (72,7\%). Dois indicadores com pior e melhor desempenho encontram-se na subdimensão Articulação com a População. $O$ indicador Plano Municipal de Saúde apresentou o pior desempenho, com 65,5\% dos municípios classificados como ruim e o indicador Relatório de Gestão apresentou o melhor desempenho, bom para 56,7\% dos municípios.

A distribuição espacial da avaliação da gestão identificou municípios com bom desempenho em todas as regiões do estado. No interior do estado observa-se maior concentração de municípios com bom desempenho nas regiões Oeste e Meio Oeste do estado, tanto na dimensão Garantia de Recursos quanto na Avaliação da Gestão. No litoral, são as regiões Nordeste e Sul que apresentam maior concentração de municípios com bom desempenho (Figura 1).

Em Santa Catarina o desempenho da gestão municipal para o planejamento em saúde esteve associado à localização geográfica $(\mathrm{p}<0,001)$. Municípios nas regiões oeste e litorânea do estado apresentaram proporcionalmente desempenho melhor do que os localizados na região central. Municípios de menor porte populacional, maior IDH e com menor percentual de investimento próprio de recursos em saúde apresentaram proporcionalmente desempenho melhor que os demais, mas tais diferenças não foram estatisticamente significantes (Tabela 3).

\section{Discussão}

O presente estudo obteve adesão de $100 \%$ dos municípios em um período relativamente curto de coleta, aspecto incomum em pesquisas com esta metodologia. A literatura refere que estudos 
Tabela 1. Matriz para emissão de juízo de valor segundo seus indicadores, subdimensões, dimensões e gestão.

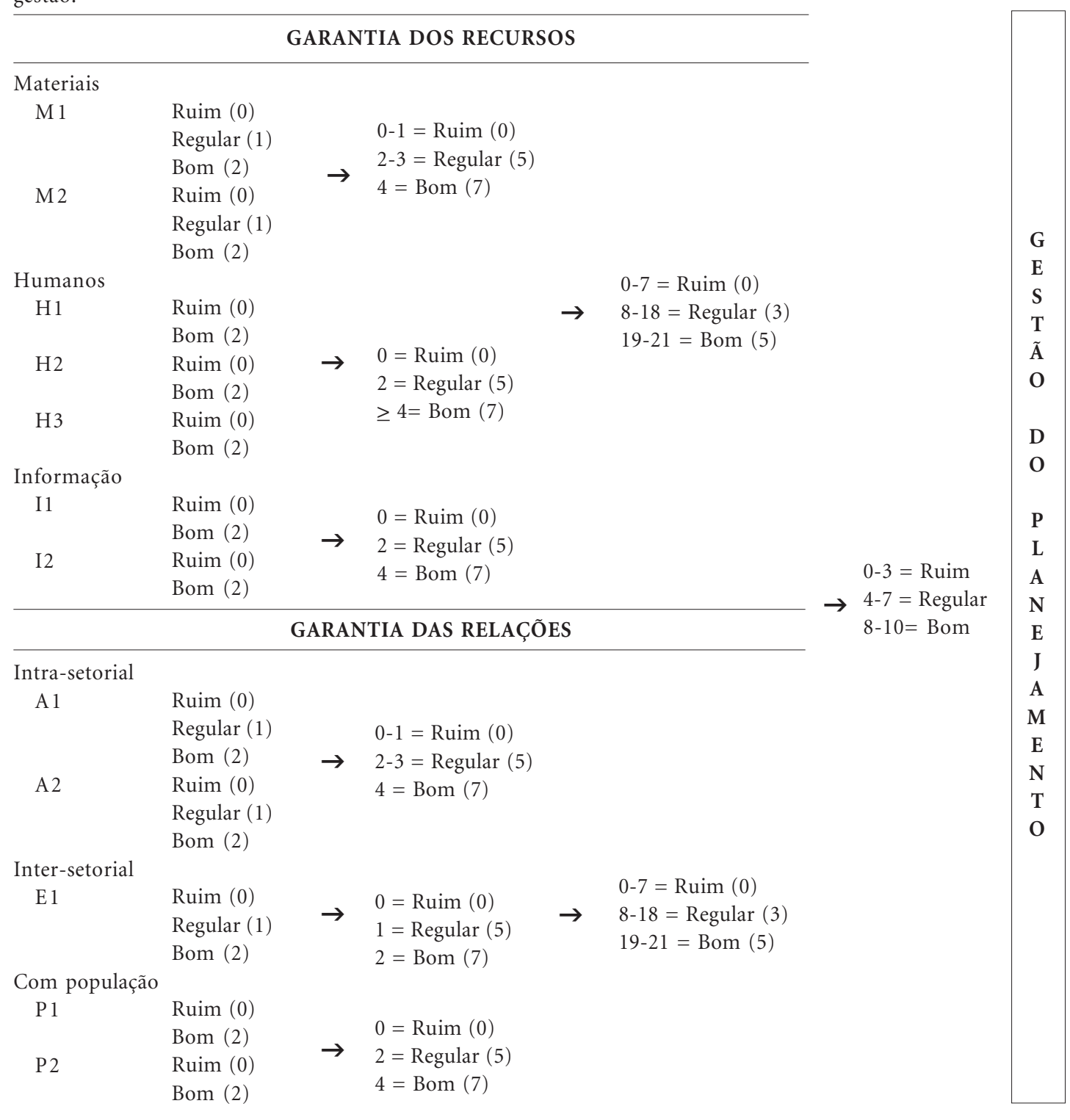

M1: Espaço Físico; M2: Apoio logístico; H1: Vínculo; H2: Permanência: H3: Qualificação; I1: Atualização de bancos; I2: Equipamento; A1: Inserção organizacional; A2: Articulação com outras áreas técnicas; E1: Articulação com outras secretarias; P1: Plano Municipal de Saúde; P2: Relatório Anual de Gestão

Fonte: Berretta et al. ${ }^{2}$

com metodologia similar apresentam uma taxa de resposta $11 \%$ abaixo dos estudos com outras metodologias ${ }^{4}$. A presença de um pesquisador à disposição dos municípios via on-line ou por telefone durante todo o período, e o fato da Secretaria de Estado da Saúde ter assumido a pesquisa como uma ação de gestão para aprimoramento do planejamento em saúde, em seu território, deve ter contribuído para este resultado. Por outro lado, o caminho da institucionalização da avaliação da atenção básica percorrido em Santa Catarina ${ }^{5}$ também deve ser considerado nesta explicação.

$\mathrm{Na}$ área do planejamento o gestor municipal apresentou melhor desempenho na garantia de aspectos estruturais, assegurando principalmente recursos de informação e de pessoal. O desempenho predominantemente regular para os recursos materiais, relacionados à qualidade do espaço físico e à presença de itens básicos para o 
Tabela 2. Número e percentual de municípios segundo classificação na avaliação da gestão municipal para o planejamento em saúde para indicadores, subdimensões, dimensão e gestão. Santa Catarina, 2010.

\begin{tabular}{|c|c|c|c|c|c|c|}
\hline \multirow{3}{*}{$\begin{array}{l}\text { DIMENSÃO } \\
\text { Subdimensão } \\
\text { Indicadores }\end{array}$} & \multicolumn{6}{|c|}{ Classificação } \\
\hline & \multicolumn{2}{|c|}{ Ruim } & \multicolumn{2}{|c|}{ Regular } & \multicolumn{2}{|c|}{ Bom } \\
\hline & $\mathbf{N}$ & $\%$ & $\mathrm{~N}$ & $\%$ & $\mathbf{N}$ & $\%$ \\
\hline GARANTIA DE RECURSOS & 19 & 6,5 & 178 & 60,8 & 96 & 32,8 \\
\hline Materiais & 78 & 26,6 & 163 & 55,6 & 53 & 18,1 \\
\hline Espaço físico & 71 & 24,2 & 152 & 51,9 & 70 & 23,9 \\
\hline Apoio logístico. & 48 & 16,4 & 135 & 46,1 & 110 & 37,5 \\
\hline Humanos & 39 & 13,3 & 111 & 37,9 & 143 & 48,8 \\
\hline Vínculo & 154 & 52,6 & - & - & 139 & 47,4 \\
\hline Permanência & 133 & 45,4 & - & - & 160 & 54,6 \\
\hline Qualificação & 154 & 52,6 & - & - & 139 & 47,4 \\
\hline Informação & 18 & 6,1 & 86 & 29,4 & 189 & 64,5 \\
\hline Bancos atualizados & 67 & 22,9 & - & - & 226 & 77,1 \\
\hline Equipamentos & 55 & 18,8 & - & - & 238 & 81,2 \\
\hline GARANTIA DAS RELAÇÕES & 37 & 12,6 & 213 & 72,7 & 43 & 14,7 \\
\hline Articulação Intrassetorial & 47 & 16 & 200 & 68,3 & 46 & 15,7 \\
\hline Inserção organização & 60 & 20,5 & 91 & 31,1 & 142 & 48,5 \\
\hline Artic. outras áreas & 28 & 9,6 & 162 & 55,3 & 103 & 35,2 \\
\hline Articulação Intersetorial & 53 & 18,1 & 157 & 53,6 & 83 & 28,3 \\
\hline Articulação com outras secretarias & 53 & 18,1 & 157 & 53,6 & 83 & 28,3 \\
\hline Articulação com População & 84 & 28,7 & 151 & 51,5 & 58 & 19,8 \\
\hline Plano Municipal de saúde & 192 & 65,5 & - & - & 101 & 34,5 \\
\hline Relatório de gestão & 127 & 43,3 & - & - & 166 & 56,7 \\
\hline EFICÁCIA DA GESTÃO & 40 & 13,7 & 140 & 47,8 & 113 & 38,6 \\
\hline
\end{tabular}
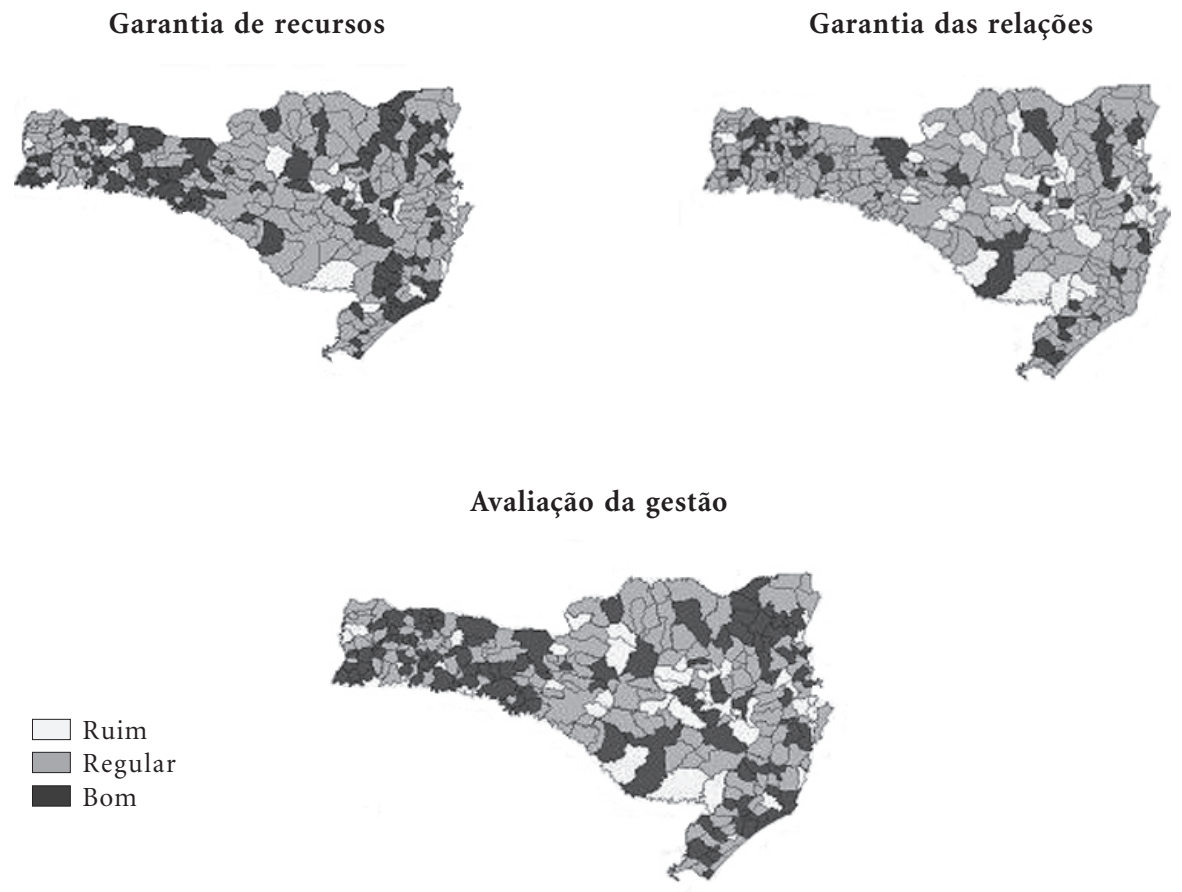

Figura 1. Análise espacial da gestão municipal para o planejamento em saúde segundo dimensões e emissão do juízo final. Santa Catarina, 2010. 
Tabela 3: Desempenho da gestão municipal para o planejamento em saúde e fatores correlatos. Santa Catarina, 2010.

\begin{tabular}{|c|c|c|c|c|c|c|}
\hline & \multicolumn{6}{|c|}{ Bom desempenho } \\
\hline & \multicolumn{2}{|c|}{ Gestão } & \multicolumn{2}{|c|}{ Recursos } & \multicolumn{2}{|c|}{ Relações } \\
\hline & $\mathbf{N}$ & $\%$ & $\mathbf{N}$ & $\%$ & $\mathbf{N}$ & $\%$ \\
\hline \multicolumn{7}{|l|}{ Localização Geográfica } \\
\hline Região Oeste $(\mathrm{n}=97)$ & 50 & 51,5 & 41 & 42,3 & 17 & 17,5 \\
\hline Região Litorânea $(\mathrm{n}=100)$ & 42 & 42,0 & 35 & 35,0 & 18 & 18,0 \\
\hline Região Central $(n=96)$ & 21 & 21,9 & 20 & 20,8 & 08 & 8,3 \\
\hline Valor de $p$ & \multicolumn{2}{|c|}{$<0,001$} & \multicolumn{2}{|c|}{0,005} & \multicolumn{2}{|c|}{0,100} \\
\hline \multicolumn{7}{|l|}{ Porte Populacional } \\
\hline Menos de 20 mil hab. $(n=172)$ & 70 & 40,7 & 60 & 34,9 & 27 & 15,7 \\
\hline De 10 a 30 mil habitantes $(n=82)$ & 32 & 39,0 & 24 & 29,3 & 13 & 15,9 \\
\hline Acima de 30 mil habitantes $(n=39)$ & 11 & 28,2 & 12 & 30,8 & 03 & 7,7 \\
\hline Valor de $p$ & \multicolumn{2}{|c|}{0,349} & \multicolumn{2}{|c|}{0,645} & \multicolumn{2}{|c|}{0,416} \\
\hline
\end{tabular}

desenvolvimento das ações de planejamento (telefone, fax, material de expediente) sugere a necessidade de políticas de incentivo para poder garantir condições físicas de trabalho na área, que podem ser preconizadas a partir dos financiamentos previstos no Pacto de Gestão. Os equipamentos de qualidade e os sistemas de informação são muito importantes no planejamento, e a atualização de bancos, que possui normativa própria, reflete a qualidade da organização desses sistemas ${ }^{6}$. O planejamento parte de um bom diagnóstico ${ }^{7}$ o qual depende de dados atualizados e confiáveis. Os dados registrados quando classificados, organizados e interpretados dentro de um contexto, geram conhecimento, propiciando uma melhor compreensão da situação de saúde. Cohn et al. ${ }^{8}$ corroboram essa reflexão ao indicarem a necessidade dos municípios superarem a forte motivação econômico-financeira para a coleta de dados e passarem a utilizá-los na geração de conhecimento que auxilie no planejamento de ações factíveis e que abriguem as necessidades reais de atendimento à saúde.

Na subdimensão Recursos Humanos o desempenho dos municípios para os indicadores vínculo de trabalho e qualificação de profissionais possibilitou demonstrar a precariedade na relação trabalhista e na política de educação permanente no setor. Mais da metade dos municípios catarinenses não atingiram os parâmetros mínimos estabelecidos de pessoal com vínculo permanente e de qualificação na área de planejamento. As exigências atuais em relação às atividades de planejamento pressupõem a necessidade de profissionais qualificados e permanentes para seu desenvolvimento. O vínculo não permanente de trabalho no serviço público contraria os princípios normativos da administração pública ${ }^{9}$. O não cumprimento desta norma pode comprometer a continuidade do processo de trabalho e até mesmo inviabilizar o cumprimento das ações planejadas, em caso de mudança de gestão. O tempo médio de permanência de um profissional na área de planejamento deve superar um período de gestão como forma de garantir a continuidade das atividades. Apesar de ter se destacado positivamente na comparação com os demais indicadores, o fato de $45,4 \%$ dos municípios apresentarem desempenho ruim no indicador permanência de profissionais, caracteriza uma alta rotatividade destes na área do planejamento. A qual é conceituada como a medida do número de trabalhadores que passa pelos postos de trabalho de uma seção, empresa, setor ou ramo, num determinado período de tempo. São apontados como fatores responsáveis pela alta rotatividade de profissionais de saúde, a insatisfação gerada por baixos salários, condições de trabalho inadequadas e formas de contratação ${ }^{10}$, portanto pode-se pensar numa correlação entre o pouco tempo de permanência dos profissionais na área de planejamento e a precariedade do vínculo apresentado pela maioria dos municípios.

Quanto à qualificação, considera-se que a formação de especialistas em planejamento ou saúde pública imprime qualidade ao desenvolvimento das ações. A análise dos indicadores vínculo e permanência evidenciou associação entre eles, mas não deles com qualificação. A concentração de Instituições de Ensino na região litorâ- 
nea favorece a formação nos municípios dessa região, mas dificulta para a maioria dos municípios de pequeno porte localizados no interior do estado. Portanto, a modalidade de formação à distância pode possibilitar a participação do maior número de profissionais da área ${ }^{11,12}$.

Além dos aspectos estruturais é preciso assegurar articulação com setores internos ou externos ao setor saúde e com representantes da sociedade civil organizada. Esta articulação busca viabilizar ações conjuntas dentro e fora do setor, essenciais à integralidade, universalidade e equidade na atenção em saúde. A maioria dos municípios catarinenses foi classificada em "regular" na dimensão de Garantia das Relações, o que sugere que a interação entre os setores de interesse para o desenvolvimento das ações de planejamento em saúde ainda é incipiente e representa um desafio que deve ser superado pelos governos locais.

O bom desempenho do indicador Relatório de Gestão como expressão de articulação com a população poderia denotar maturidade do gestor quanto à necessidade da participação da população nas decisões da gestão, mas deve-se considerar o fato de a aprovação desse instrumento de gestão, além de obrigatória, ser condicionante a vários aportes financeiros. Além disso, observou-se resultado ruim no indicador Plano de saúde, denotando que a articulação da gestão com a população, mesmo constituindo um princípio organizativo do Sistema, não é realizada pela maioria dos municípios catarinenses.

A distribuição espacial dos municípios com classificações melhores na avaliação geral foi bastante homogênea, tanto que foram 113 municí- pios (38,6\% do total) com conceito "bom", onde residem aproximadamente $30 \%$ da população do estado, para a dimensão Garantia de Recursos, 96 municípios $(32,8 \%)$ tiveram classificação boa, correspondendo a $37 \%$ da população do estado. A pior condição foi observada na dimensão Garantia das Relações, com 43 municípios (14,7\%) bons, correspondendo a apenas $23 \%$ da população do estado. Esse baixo número de municípios com boa Garantia das Relações é muito preocupante, uma vez que apenas aspectos estruturais não garantem legitimidade ao planejamento em saúde. São necessárias negociações, articulações e pactos para garantir que as propostas sejam viabilizadas e tenham continuidade.

Localização geográfica e porte populacional foram variáveis investigadas quanto a possíveis associações com as melhores classificações na avaliação. A região central do estado apresentou os menores percentuais de bom desempenho, e esse resultado é coerente com as análises dos indicadores de saúde de diversos municípios dessa região ${ }^{13}$. A análise da associação com o porte, apesar de não evidenciar diferença significativa, apresenta um percentual expressivamente baixo $(7,7 \%)$ para municípios maiores com bom desempenho da Garantia das Relações. Essa dimensão denota a preocupação em consolidar o planejamento com ações que estabeleçam parcerias internas e externas ao setor saúde, e o pequeno número de municípios maiores bem posicionados nesse aspecto é ainda mais preocupante, uma vez que neles residem as maiores necessidades de pactos que garantam boa consecução das ações de planejamento. 


\section{Colaboradores}

JT Lacerda, MCM Calvo, IQ Berretta e AMB Ortiga participaram igualmente de todas as etapas de elaboração do artigo.

\section{Referências}

1. Brasil. Ministério da Saúde (MS). Sistema de planejamento do SUS: uma construção coletiva. Brasília: Ministério da Saúde (MS); 2006. (Série Cadernos de Planejamento)

2. Berretta IQ, Lacerda JT; Calvo MCM. Modelo de avaliação da gestão municipal para o planejamento em saúde. Cad Saude Publica 2011; 27(11):2143-2154.

3. Brasil. Ministério da Saúde (MS). Pactos pela Vida e de Gestão: Normas e Manuais Técnicos. Brasília: Ministério da Saúde (MS); 2006. (Série Pactos pela Saúde, V. 2)

4. Fan W, Yan Z. Factors affecting response rates of the web survey: A systematic review. Computers in Human Behavior 2010; 26(2):132-139.

5. Felisberto E, Freese E, Bezerra LCA, Alves CKA, Samico I. Análise da sustentabilidade de uma política de avaliação: o caso da atenção básica no Brasil. Cad Saude Publica 2010; 26(6):1079-1095.

6. Brasil. Ministério da Saúde (MS). Sistema de Planejamento do SUS: uma construção coletiva. Brasília: Ministério da Saúde (MS); 2006. (Série Cadernos de Planejamento, V. 1).

7. Rivera U, Matus C, Testa M. Planejamento e programação em saúde um enfoque metodológico. São Paulo: Cortez; 1989.

8. Cohn A, Westphal MF, Elias PE. Informação e decisão política em saúde. Rev Saude Publica 2005; 39(1):114-121.

9. Brasil. Presidência da República. Casa Civil. Constituição da República Federativa do Brasil de 1988. Diário Oficial da União 1988; 6 out.

10. Campos CVA, Malik AM. Satisfação no trabalho e rotatividade dos médicos do programa de saúde da família. Rev. Adm. Pública 2008; 42(2):347-368.

11. Brasil. Ministério da Saúde (MS). Portaria no 561, de 16 de março de 2006. Institui, no Âmbito do Ministério da Saúde, a Comissão Permanente de Telessaúde. Diário Oficial da União 2006; 17 mar.

12. Melo TM, Alvarenga KF, Blasca WQ, Taga MFL. Capacitação de agentes comunitários de saúde em saúde auditiva: efetividade da videoconferência. PróFono R. Atual. Cient. 2010, 22(2):139-144.

13. Santa Catarina. Secretaria de Estado da Saúde. Gerência de Planejamento do SUS. Plano estadual de saúde. Florianópolis: IOESC; 2007.

Artigo apresentado em 17/10/2011

Aprovado em 13/11/2011

Versão final apresentada em 04/01/2012 